Preprint typeset in JHEP style - HYPER VERSION

Liverpool Preprint: LTH 662

\title{
The effect of sea quarks on the mass of the charm quark from lattice QCD
}

\section{UKQCD Collaboration}

\author{
A. Dougall \\ Department of Physics and Astronomy, University of Glasgow, Glasgow, G12 8QQ UK. \\ C.M. Maynard \\ School of Physics, JCMB, Kings Buildings, University of Edinburgh, Edinburgh, EH9 \\ 3JZ, UK.

\section{McNeile} \\ Theoretical Physics Division, Dept. of Mathematical Sciences, University of Liverpool, \\ Liverpool L69 3BX, UK
}

\begin{abstract}
We compute the mass of the charm quark using both quenched and dynamical lattice QCD calculations. We examine the effects of mass dependent lattice artifacts by comparing two different formalisms for the heavy quarks. We take the continuum limit of the charm mass in quenched QCD by extrapolating from three different lattice spacings. At a fixed lattice spacing, the mass of the charm quark is compared between quenched QCD and dynamical QCD with a sea quark mass around strange. In the continuum limit of quenched QCD, we find $m_{c}\left(m_{c}\right)=1.29(7)(13) \mathrm{GeV}$. No evidence was seen for unquenching.
\end{abstract}

KEYWORDS: Lattice QCD. 


\section{Contents}

1. Introduction 1

2. Details of the calculation 3

3. Definitions of the quark mass

4. Perturbative matching 6

4.1 Quark mass renormalisation factors

4.2 Discussion of non-perturbative matching 8

4.3 Evolving the quark mass to the charm quark mass 11

4.4 Coupling prescription 11

5. Interpolations in quark mass 11

6. Results 12

6.1 Examples of data fits 13

6.2 Results at fixed lattice spacing 14

6.3 Estimating the final systematic error

7. Conclusions 19

8. Acknowledgements 21

\section{Introduction}

The mass of the charm quark is a fundamental parameter of the Standard Model (SM), and yet its value is rather imprecise. The Particle Data Group [1] quote

$$
1.15<\bar{m}_{\text {charm }}^{\overline{\mathrm{MS}}}\left(\bar{m}_{\text {charm }}\right)<1.35 \mathrm{GeV}
$$

This is to be contrasted with the more precise value of the mass of the $D_{s}$ :

$$
m_{D s}=1.9695(5) \mathrm{GeV}
$$

from experiment. The problem is of course that quarks are confined hence their mass can never be directly measured. The imprecise value of the charm mass is just a reflection on how hard it is to solve QCD from first principles.

The value of the charm mass is important for phenomenology. For instance the uncertainty in the charm quark mass is a big source of uncertainty in the production of charm 
from DIS processes at HERA [2]. Some models of physics beyond the standard model predict relations between various parameters such as the quark masses. The mass of the charm quark is also potentially important for understanding kaon decays [3]. See the review article [4] for a comprehensive review of the mass of the charm quark.

Lattice QCD can determine the hadron spectrum for a given quark mass. This can then be compared to experiment, and the quark mass tuned until the spectrum produced matches the experimental one. In practice the systematic uncertainties from the finite lattice spacing and too heavy sea quarks make this a non-trivial task in general. There have been many calculations of the mass of the charm quark from quenched QCD [5, 6, 7, 8, 9, 10, however there has not been an estimate of the error due ignoring the effect of virtual quark anti-quark pairs on the mass of the charm quark.

What effect the sea quarks has on the mass of the charm quark is very difficult to estimate without simulating full QCD. However, using the running of the quark mass, in the quenched and $N_{F}=2$ world, Mackenzie [11, 12 estimated that sea quarks could reduce the light quark masses by $10-20 \%$. There have been claims that the light quark masses in unquenched QCD are significantly different to their values in quenched QCD. The CPPACS collaboration [13] found that light quark masses with sea quarks were $25 \%$ lower than the quenched results. The recent computation, undertaken jointly by the HPQCD, MILC and UKQCD collaborations, of the strange quark mass is also significantly less than the result in quenched QCD [14, 15]. All the above calculations used perturbative renormalisation.

Recently, there have been a number of two flavour unquenched lattice QCD calculations, using Wilson or clover fermions, that have found that the strange quark mass is consistent with the value from quenched QCD [16, 17, 18, 19]. These new calculations use sea quarks with masses above a third of the strange quark mass, but do consistently use non-perturbative renormalisation techniques. The use of renormalisation factors, to two loop accuracy, by the HPQCD and UKQCD collaborations [15] in the analysis of data from the lattice calculations that use improved staggered fermions moved the value of the strange quark mass closer to the quenched value, but still remained below it. The sea quarks used in the lattice calculations with Wilson or clover fermions [16, 17, 18, 19] are much heavier that those used by the HPQCD, MILC and UKQCD collaborations 14, 15]. The situation is not clear at the moment, but the introduction of sea quarks into a lattice QCD calculation could reduce the value of the strange quark mass by value between $0 \%$ and $10 \%$.

Only small differences have been found between the mass of the $b$ quark in quenched and unquenched QCD 20, 21, 22, 23]. The results for quark masses from the lattice have been reviewed by Lubicz [24], Wittig [25], and Rakow [26].

In this paper we make the first attempt to study the effect of sea quarks on the charm quark mass. Naively we would expect that the size of this effect would lie between that of the effect for strange and bottom quark masses.

The mass of the charm quark is sizable in units of the lattice spacing that are computationally feasible for unquenched calculations, hence the errors from the finite size of the lattice spacing are of great concern in this paper. There are a number of different lattice 


\begin{tabular}{ccccc}
$\left(\beta, \kappa_{\text {sea }}\right)$ & Volume & $a^{-1} \mathrm{GeV} r_{0}=0.5 \mathrm{fm}$ & $a^{-1} \mathrm{GeV} r_{0}=0.55 \mathrm{fm}$ & number of configurations \\
\hline$(6.2,0)$ & $24^{3} \times 48$ & 2.913 & 2.648 & 216 \\
$(6.0,0)$ & $16^{3} \times 48$ & 2.119 & 1.926 & 302 \\
$(5.93,0)$ & $16^{3} \times 32$ & 1.860 & 1.691 & 278 \\
$(5.2,0.1350)$ & $16^{3} \times 32$ & 1.876 & 1.706 & 395
\end{tabular}

Table 1: Ensemble of gauge configurations. $\kappa_{\text {sea }}=0$ denotes a quenched ensemble.

formalisms (recently reviewed by Kronfeld [27], Hashimoto and Onogi [28]), so there are a number of different ways of organising the calculations.

Following the introduction, in section 2 we discuss the parameters of the lattice calculations. The different definitions of the quark masses are then outlined in section 3 , followed by section 1 , in which we describe the perturbative matching factors between the lattice data and the $\overline{\mathrm{MS}}$ scheme. In section $\mathrm{f}$ we discuss the methods used to interpolate from the quark masses at which the calculation was performed to the physical points. The final sections detail our results and conclusions.

\section{Details of the calculation}

The gauge fields were generated with Wilson's plaquette action and the quarks with the clover action, where the coefficient of the Sheikholeslami-Wohlert term, $c_{S W}$, has been determined non-perturbatively (NP). In this way the leading discretisation effects of the lattice are reduced from $\mathcal{O}(a)$ to $\mathcal{O}\left(a^{2}\right)$ for hadron masses, where $a$ is the lattice spacing. Whilst this does not guarantee that lattice artifacts are smaller, the continuum limit is approached as a function of $a^{2}$.

Hadron correlation functions were computed on three ensembles of gauge field configurations in the quenched approximation, $\beta=\{6.2,6.0,5.93\}$ and one ensemble of configurations with the sea quarks, $\left\{\beta=5.2, \kappa_{\text {sea }}=0.135\right\}$. The values of the gauge coupling and quark mass of this ensemble were chosen so that the lattice spacing is matched to the coarsest quenched ensemble. The parameters of the lattice calculations are presented in table 1. We will use the $\beta$ value to distinguish each ensemble. The UKQCD collaboration has previously presented results and full details of the calculation for the light hadron spectrum [29] and heavy-light spectrum and currents [30] on the finest two of the three quenched ensembles, and the light hadron spectrum and currents on the matched quenched and sea quark ensembles [31]. We have already presented results for the heavy-light spectrum on these four ensembles in [32]. Some of the charmonium mass spectrum from the unquenched data set has been reported in [33]. In this work we extend the analysis to the correlators necessary to define the mass of the charm quark.

For the $\beta=6.0$ and $\beta=6.2$ data sets, single or double exponential fits were made to the smeared source and local sink correlators. The gauge invariant smearing formalism of Boyle was used [34] with the parameters in [30]. For the $\beta=5.2$ and $\beta=5.93$ data sets, we fitted a variational multi-exponential fitting model to a 2 by 2 matrix of correlators made from a basis of local and fuzzed operators [35] . 


\section{Definitions of the quark mass}

There are a number of different ways of calculating a bare lattice quark mass from the parameters in a lattice QCD calculation. The different definitions of the quark mass have different $\mathcal{O}(a)$ effects. This is clearly seen in quenched calculations where a continuum limit is required for the two definitions to agree [6, 36]. In this section we discuss various improved definitions of the quark mass that should have reduced lattice spacing dependence. The connection between the quark masses from the lattice and those in the continuum $\overline{\mathrm{MS}}$ scheme is discussed in section 6 . In the following we shall use lower cases for the quark masses and upper cases for the meson masses.

One definition of the quark masses is from the Vector Ward identity

$$
a m_{0}=\frac{1}{2}\left(\frac{1}{\kappa}-\frac{1}{\kappa_{\text {crit }}}\right)
$$

In the mass independent renormalisation scheme of the ALPHA collaboration [37, 38, 39] the vector definition of the quark mass is $\mathcal{O}(a)$ improved using

$$
a m_{0}^{I}=a m_{0}\left(1+b_{m} a m_{0}\right)
$$

The quark mass can also be defined from the Axial Ward Identity. This is often known as the PCAC mass. The axial current and pseudo-scalar density are defined as

$$
\begin{aligned}
A_{\mu}(x) & =\bar{\psi}_{i}(x) \gamma_{5} \gamma_{\mu} \psi_{j}(x) \\
P(x) & =\bar{\psi}_{i}(x) \gamma_{5} \psi_{j}(x)
\end{aligned}
$$

Although both the $A_{\mu}$ and $P$ operators depend on the flavor indices $i$ and $j$, for simplicity we suppress the explicit dependence. The axial current can be improved according to 40

$$
A_{\mu}^{\mathrm{I}}(x)=A_{\mu}(x)+a c_{A} \partial_{\mu} P(x)
$$

These currents are then renormalised as

$$
J^{R}=Z_{J}\left(1+b_{J}\left(a m_{i j}\right)\right) J^{\mathrm{I}}
$$

where $J$ is either $A$ or $P$. The bare PCAC quark mass can then be defined in terms of correlation functions of the bare currents

$$
a m_{p c a c, i j}=\frac{\sum_{\vec{x}}\left\langle\partial_{4} A_{4}^{\mathrm{I}}(x) P(0)\right\rangle}{2 \sum_{\vec{x}}\left\langle P^{\mathrm{I}}(x) P(0)\right\rangle}
$$

and the renormalised quark mass is given by

$$
a m_{p c a c, i j}^{I}=\left[1+\left(b_{A}-b_{p}\right) a m_{0, i j}\right] a m_{p c a c, i j}
$$

where the quark mass, $m_{q, i j}$, is given by

$$
a m_{q, i}+a m_{q, j}=2 a m_{q, i j}
$$


for $q$ either 0 or pcac.

The values of the improvement coefficients $b_{J}$ and $c_{J}$ are known to one loop in perturbation theory [40, 41]. The improvement coefficients are also known non-perturbatively in quenched QCD for $\beta>6.0$. The $c_{A}$ coefficient has recently been computed in unquenched QCD with 2 flavours of clover fermions [42]. We discuss the use of the non-perturbative improvement factors in section 1 .

To make the best use of the existing one loop results we use the tadpole improved formalism of Lepage and Mackenzie [43]. In this formalism the normalisation of the quark field changes,

$$
\sqrt{2 \kappa} \psi \rightarrow \sqrt{2 u_{0} \kappa} \psi
$$

where $u_{0}$ is defined by

$$
u_{0}=\left\langle\frac{1}{3} \operatorname{tr}\left[U_{\mu \nu}(x)\right]\right\rangle^{\frac{1}{4}}
$$

Following Bhattacharya et al. [44, 45], the expressions for the coefficients determined by Sint and Weisz can be re-written to form the tadpole improved expressions

$$
\begin{aligned}
c_{A} & =-0.0952 \alpha_{s} \\
b_{P} & =\frac{1}{u_{0}}\left(1+0.8763 \alpha_{s}\right) \\
b_{A} & =\frac{1}{u_{0}}\left(1+0.8646 \alpha_{s}\right) \\
b_{m} & =\frac{1}{u_{0}}\left(-\frac{1}{2}-0.685 \alpha_{s}\right)
\end{aligned}
$$

We also investigated other heavy quark formalisms that claim to have a smaller lattice spacing dependence. For heavy quarks with the improvement coefficients determined from one-loop perturbation theory the leading cut-off effects will be $\mathcal{O}\left(\alpha_{s} a m\right)$. In particular, for the dynamical ensemble, for which we cannot take the continuum limit, the lattice space is coarse $(\mathcal{O}(a m) \sim 0.6)$. An effective field theory approach is the FNAL method [46] which supposes the dominance of $\mathcal{O}\left((a m)^{n}\right)$ cut-off effects over $\mathcal{O}\left((a p)^{2}\right)$ effects.

The lattice distorts the dispersion relation for a particle in the following way

$$
E^{2}=M_{1}^{2}+\frac{M_{1}}{M_{2}} p^{2}+\mathcal{O}\left(p^{4}\right)
$$

where $M_{1}$ is the rest mass and $M_{2}$ is the kinetic mass, defined by

$$
\frac{1}{M_{2}}=\left.\frac{\partial^{2} E}{\partial p_{k}^{2}}\right|_{p=0}
$$

An example of the energies of a typical data set fitted with both the continuum and FNAL dispersion relations is given in figure 1. 1 .

In the Fermilab method it is the kinetic mass that is important for the dynamics of heavyheavy and heavy-light bound states. The deviation of $M_{1}$ from $M_{2}$ can give a measure of mass dependent cut-off effects. The quark mass, $m_{1}$, is defined as

$$
a m_{1}=\log \left(1+a m_{0}\right)
$$




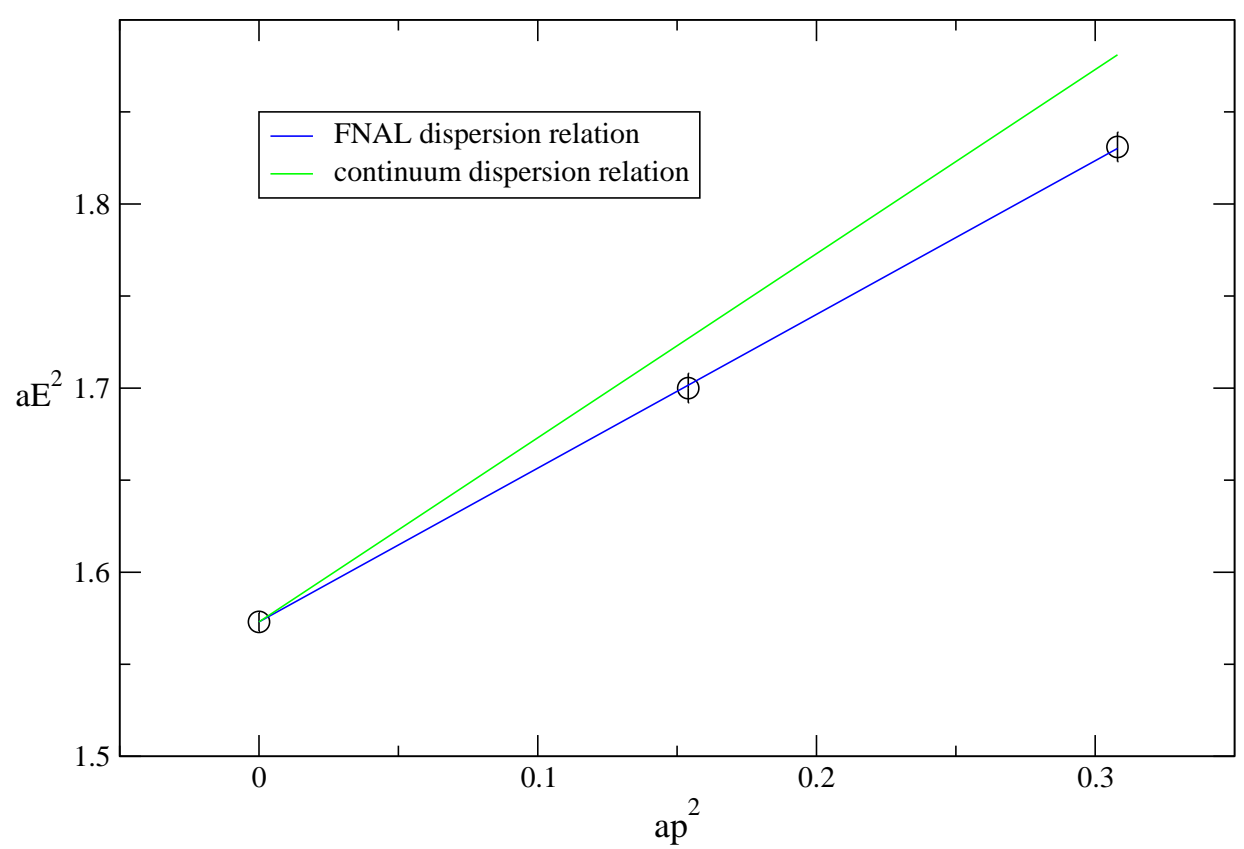

Figure 1: Example of the energies for a typical data set $\left(\beta=5.2, \kappa_{\mathrm{H}}=0.1130\right.$ and $\left.\kappa_{\mathrm{L}}=0.1340\right)$, fitted using the continuum and FNAL dispersion relations.

where $m_{0}$ is defined in equation (3.1). Note the similarity of $m_{1}$ to the unrenormalised RGI mass in equation (3.2), to $\mathcal{O}\left(a^{2}\right)$ at least, as

$$
\log (1+a m)=a m-\frac{1}{2}(a m)^{2}+\frac{1}{3}(a m)^{3} \cdots
$$

and $b_{m}=-\frac{1}{2}$ at tree-level. This is the tree-level expression (in $g^{2}$ ) for the quark mass, to all orders in am. A perturbative definition of the kinetic quark mass is given by

$$
a m_{2}\left(a m_{1}\right)=\frac{e^{a m_{1}} \sinh \left(a m_{1}\right)}{1+\sinh \left(a m_{1}\right)}
$$

The quark masses, $m_{1}$ and $m_{2}$ can be used to get a perturbative definition of the hadron kinetic mass

$$
a M_{2}^{P T}=a M_{1}+\left(a m_{2}-a m_{1}\right)
$$

In this work we study equation 3.20 , both at tree level and at one loop in perturbation theory.

\section{Perturbative matching}

We briefly describe the formalism required to extract the quark mass in the $\overline{\mathrm{MS}}$ scheme at a specific reference scale. Most of the formalism is taken from [47, 36, 48]. We only use perturbative matching. Non-perturbative matching is discussed in section 4.2 . 


\subsection{Quark mass renormalisation factors}

To extract the quark mass in $\overline{\mathrm{MS}}$ we use the one loop matching factor

$$
m_{0}^{\overline{\mathrm{MS}}}(\mu)=Z_{m}(a \mu) X m_{0}^{I}(a)
$$

where $Z_{m}(a \mu)$ is the perturbative matching factor, $X$ is tadpole improvement factor, and $m_{V I}(a)$ is the bare lattice quark mass.

$$
Z_{m}(a \mu)=1-\frac{\alpha(\mu)_{s}}{4 \pi}\left(8 \ln (\mu a)-\left(C_{M}-t a d\right)\right)
$$

The QCDSF collaboration have published expressions for $C_{M}$ as a function of the coefficient of the clover term $c_{s w} 48$.

$$
C_{M}=\frac{4}{3}\left(12.952+7.738 c_{s w}-1.380 c_{s w}^{2}\right)
$$

The numerical value of $C_{M}=25.758$ for $C_{S W}=1$. At one loop it is consistent to use the one loop value for $c_{S W}$. Recently, the QCDSF collaboration have claimed substantial differences between the renormalisation of the vector definition of the quark mass depending on whether the singlet or non-singlet estimate of the $Z_{m}$ factor is used [16]. In this calculation there are no charm quarks in the sea, hence only the standard non-singlet renormalisation factor is used for the vector quark mass. The ALPHA collaboration compute the connection between the quark mass on the lattice and the renormalisation group invariant mass [39] using a non-perturbative procedure.

The tadpole improved [43, 36] value for $Z_{m}$, based on $X=8 \kappa_{\text {crit }}$, uses tad $=10.66$. For the non-tadpole improved case: $X=1$ and $\operatorname{tad}=0$.

At one loop, in some sense the scale $\mu$ in equation 4.1 is a free parameter. In principle no physical prediction should depend on the value of $\mu$. The dependence on $\mu$ is reduced as the number of loops is increased. Reasonable choices for $\mu$ lie in the range from $1 / a$ to $\pi / a$. The "best guess scale" for the $\mu$ (called $q \star$ ), that attempts to minimise higher order corrections, can in principle be computed using the formalism described by Lepage and Mackenzie [43]. There has been a recent calculation of the $q \star$ for many of the perturbative expressions required for the PCAC mass 49.

The connection between $m^{\overline{\mathrm{MS}}}$ and the PCAC mass is in 4.4 .

$$
m_{p c a c}^{\overline{\mathrm{MS}}}(\mu)=\frac{Z_{A}}{Z_{P}} m_{p c a c}^{I}
$$

The tadpole improved matching factors for the axial and pseudo-scalar operators are 444]:

$$
Z_{P}(\mu)=u_{0}\left(1+\alpha_{s}\left(\frac{1}{4 \pi} \log (a \mu)^{2}-1.328\right)\right)
$$

and

$$
Z_{A}=u_{0}\left(1-0.416 \alpha_{s}\right)
$$


The Fermilab group [50] have computed the connection between the lattice quark mass and the pole quark mass to all orders in $m$ at one loop order in the expansion of the coupling.

$$
m_{1}=m_{1}^{(0)}+g^{2} Z_{m_{1}}^{(1)} \tanh m_{1}^{(0)}
$$

where

$$
Z_{m_{1}}^{(1)}=z_{m_{1}}^{(1)} e^{-m_{1}^{(0)}} \cosh m_{1}^{(0)}\left(p_{A_{0}}^{n}\left(m_{1}^{(0)}\right) A_{P V}^{1}\left(\sinh \left(m_{1}^{(0)}\right)\right)-p_{C}^{n}\left(m_{1}^{(0)}\right) B_{P V}^{1}\left(\sinh \left(m_{1}^{(0)}\right)\right)\right)
$$

The $z_{m_{1}}^{(1)}$ factor is a function of the mass and the clover coefficient that can be reconstructed from the coefficients of the Chebyshev polynomials [50]. The functions $A_{P V}, B_{P V}, p_{A}$ and $p_{C}$ are functions explicitly quoted in the Fermilab paper [50]. In the limit $m_{1} \rightarrow 0$,

$$
Z_{m_{1}}^{(1)}=0.143+0.0653 c_{s w}-0.0116 c_{s w}^{2}-\frac{1}{4 \pi^{2}} \log \left(m_{1}^{(0)}\right)^{2}
$$

This is the lattice part of the matching factor above. The $Z_{m_{1}}^{(1)}$ factor contains the $b_{m}$ factor of Sint and Weisz [11]. In the static (infinite mass) limit $m_{1}^{(1)}(\infty)=0.168 g^{2}$ [51.

The one loop expression for the $m_{2}$ mass is

$$
m_{2}^{(1)}=m_{2}\left(m_{1}^{0}+g^{2} m_{1}^{0}\right)\left(1+g^{2} Z_{m_{2}^{(1)}}\right)
$$

where the function $m_{2}$ is defined in equation 3.19. The function $Z_{M_{2}^{1}}$ is a function of $m_{1}$ in the paper by the Fermilab group [50].

The tadpole improved definition of the $m_{1}$ mass is

$$
\hat{m}_{1}^{1}=m_{1}^{(1)}+\frac{\hat{M}_{0}}{1+\hat{M}_{0}} u_{0}^{1}
$$

where $u_{0}^{1}=1 / 12$.

The $m_{1}$ and $m_{2}$ mass definitions advocated by the Fermilab group are the lattice part of the matching. To convert the results to the $\overline{\mathrm{MS}}$ scheme, the lattice results have the log term subtracted from equation 4.9 and the results are multiplied by $Z_{\mathrm{FNAL}->\overline{\mathrm{MS}}}(\mu)$.

$$
Z_{\mathrm{FNAL}->\overline{\mathrm{MS}}}(\mu)=1-\frac{\alpha_{s}(\mu)}{3 \pi}(4+6 \log (\mu a))
$$

The importance of subtracting the log term in equations of the form 4.9 has been stressed by Groote and Shigemitsu [52]. This is equivalent to the continuum matching factor used by Davies and Thacker [53] and so the matching factor agrees with that in equation 4.2. In our early presentations [54, 55] we did not subtract the log term from equation 4.9 and only used the matching factor for the pole mass to the $\overline{\mathrm{MS}}$ scheme.

\subsection{Discussion of non-perturbative matching}

So far we have only discussed the use of matching factors to one loop in perturbation theory. There are a number of elegant numerical formalisms that can compute renormalisation factors non-perturbatively (see the reviews [56, 57, 58]). These methods promise 
a non-perturbative matching with an accuracy limited by continuum calculations that are usually known to at least two loops. There is also a program of research into computing perturbative matching factors, between the lattice and continuum QCD, to two loop accuracy [59].

A particularly nice example of the power of non-perturbative matching was the computation of the mass of the charm quark in quenched QCD by Rolf and Sint [6]. The consistent use of the non-perturbative factors from the ALPHA collaboration coupled with a controlled continuum extrapolation produced a very precise value for the charm mass in quenched QCD.

There are now many non-perturbative estimates for matching factors from unquenched QCD with clover fermions [60, 61, 62, 16, 42, 63, 18]. However, at the lattice spacing of our unquenched data it is not clear that non-perturbative renormalisation factors should automatically be used, unless the data is part of a consistent continuum extrapolation.

In their calculation of the charm mass in quenched QCD, Rolf and Sint [6] used improved coefficients and renormalisation factors determined non-perturbatively by the ALPHA collaboration in quenched QCD. At non-zero lattice spacing there were significant differences between the vector and PCAC quark masses, that extrapolated to zero as the continuum limit was taken. Rolf and Sint performed their lattice calculations with lattice spacings in the range 0.1 to $0.05 \mathrm{fm}$.

The unquenched data used in this work is at a fixed lattice spacing of $0.1 \mathrm{fm}$. At the moment it is computationally prohibitive, to do unquenched calculations with light sea quark masses and clover fermions, with a lattice spacing of $0.05 \mathrm{fm}$, using existing algorithms and computers [64, 65].

The non-perturbative estimates of improvement and matching factors can make the $O\left(a^{2}\right)$ corrections to the quark sizable. There is an $O(a)$ ambiguity to the renormalisation condition used for the non-perturbative estimate of improvement coefficient or matching factor. Different conditions can produce different results at non-zero lattice spacing, but they will agree in the continuum limit.

It has been observed that there is a large $\mathcal{O}(a)$ ambiguity in the coefficient $c_{A} 44$, 45]. This induces an $\mathcal{O}\left(a^{2}\right)$ ambiguity into the currents. This was noted because of the disagreement between the perturbative and non-perturbative estimate of $c_{A}$ at a lattice spacing of $0.1 \mathrm{fm}$ in quenched QCD.

The ALPHA collaboration have also found an $\mathcal{O}(a)$ lattice spacing ambiguity in the coefficients $b_{A}-b_{P}$ [66]. Provided that a consistent definition of the coefficients is used at each lattice spacing, the continuum will be approached smoothly, and the different definitions should have the same continuum limit. A discussion of the effect of different determinations of the improvement coefficients can be found in [30]. In particular, a naive comparison of the continuum limit of the quenched decay constant $f_{K}$, with that obtained using the ALPHA determinations [67], using different determinations of the improvement coefficients suggests that this is indeed the case.

In quenched QCD, the tadpole improved formalism has been extensively compared against the non-perturbative results 49 . A difference of $4 \%$ between the non-perturbative estimate and improved perturbative estimate was claimed. For our unquenched data at $\beta=$ 
5.2 , we saw a $10 \%$ difference between the tadpole improved estimate of $Z_{A}$ and the recent non-perturbative estimate [63]. Other groups have claimed to see systematic differences between using perturbative and non-perturbative renormalisation factors [16, 17, 19].

All current estimates of non-perturbative matching factors are done at leading order in the quark mass. At the lattice spacings we work at, the $O(a m)$ terms are not small. In figure 2 the various renormalised quark masses are plotted. The coupling for $\beta=6.0$ is used. The renormalisation factors are expanded in both quark mass and coupling. The ALPHA formalism only treats the quark mass renormalisation to leading order. A large deviation of the FNAL renormalised quark masses from the masses renormalised using the ALPHA method would show that a one loop renormalisation factor with all orders in the quark mass could be closer to the continuum result, than using the ALPHA analysis, even with non-perturbative matching, at fixed lattice spacing. Of course the ALPHA renormalisation method is better than the FNAL renormalisation as the lattice spacing is reduced, but this may not be computationally feasible. There have been attempts to develop a non-perturbative definition of the FNAL formalism 668, 69].

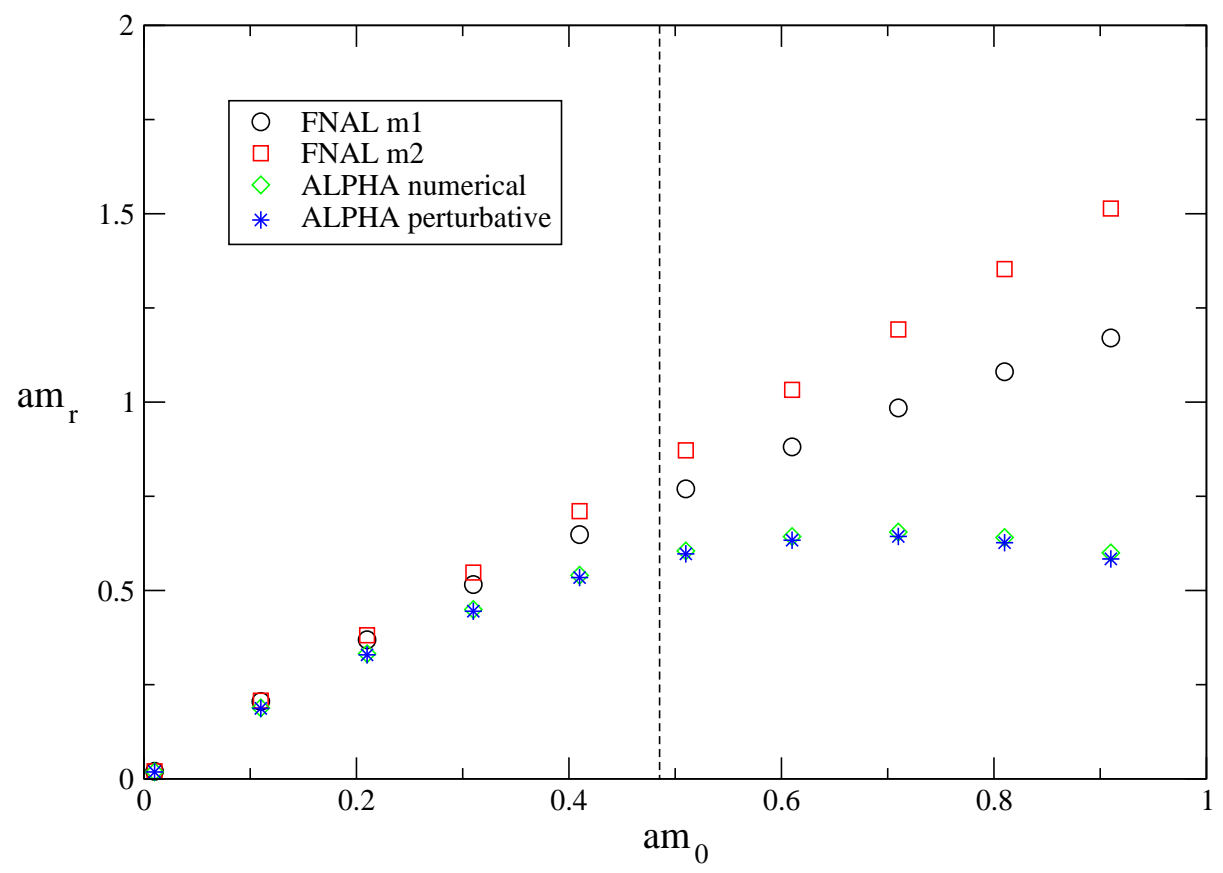

Figure 2: Renormalised group invariant quark mass as a function of the vector quark mass at $\beta=$ 6.0. The bursts are the FNAL renormalisation, the diamonds are the renormalised mass from the numerical calculations of ALPHA [6], and the squares are the perturbatively renormalised masses from the perturbative expressions in the ALPHA formalism.

A naive application of the ALPHA formulation at a fixed lattice spacing can be problematic for heavy quarks. For example in figure 2 the renormalisation factors from ALPHA bend over at masses $a m \sim 0.6$. These quark masses are too heavy for the use of $O(a)$ im- 
provement at this lattice spacing. If calculations at quark masses larger than the charm mass are required, then they should be done at a finer lattice spacing. An alternative strategy is do a heavy quark interpolation in the continuum limit [70].

Although Rolf and Sint have demonstrated that the ALPHA formulation can be used to compute the mass of the quark charm using data with lattice spacings at and finer than $0.1 \mathrm{fm}$ [6], the lattice spacing errors at $0.1 \mathrm{fm}$ are not small. Given the high computational cost of reducing the lattice spacing errors in unquenched lattice QCD calculations, we feel that it is more useful to use tadpole improved perturbation theory to one loop and investigate the FNAL formalism for this data set. This should give a result closer to the continuum limit. As the lattice spacing is reduced in unquenched calculations, then the non-perturbative renormalisation will be crucial in producing results with high accuracy.

\subsection{Evolving the quark mass to the charm quark mass}

The matching of the quark mass in the lattice scheme to the quark mass in the $\overline{\mathrm{MS}}$ scheme produces the mass at the scale $\mu$, where $\mu$ is chosen, or guessed, to minimise the higher loop corrections to the perturbative matching factor. It is conventional [1] to evolve the quark masses from the scale $\mu$ to a standard reference scale of $m_{c} \mathrm{GeV}$. This is sometimes known as the scale invariant mass.

The evolution is done using the solution of the renormalisation group equation

$$
\mu^{2} \frac{d}{d \mu^{2}} m^{\left(n_{f}\right)}(\mu)=\gamma_{m}^{n_{f}} m^{\left(n_{f}\right)}(\mu)
$$

given by

$$
\frac{m(\mu)}{m\left(\mu_{0}\right)}=\frac{c\left(\alpha_{s}(\mu) / \pi\right)}{c\left(\alpha_{s}\left(\mu_{0}\right) / \pi\right)}
$$

The anomalous dimension $\gamma_{m}^{n_{f}}$ is known to four loop order. We use the RunDec 71 mathematica package to do the evolution.

\subsection{Coupling prescription}

For the perturbative matching a choice of coupling is required, or equivalently a choice of $\Lambda_{Q C D}$. We use the $\Lambda_{M S}$ computed on the same data set [72]. This allows us to use a consistent coupling in all stages of the calculation. This is sometimes known as "horizontal matching" [36, 73]. (The $\beta=5.95$ result is used for the matched quenched data). These values are also partially quenched. We consistently use $n_{f}=2(0)$ in all the perturbative expressions for the dynamical (quenched) data set. The couplings used are presented in table 2. The coupling $\alpha \overline{\mathrm{MS}}(q)$ was calculated at any scale using the standard four loop evolution equation [71].

\section{Interpolations in quark mass}

We determined the heavy-light hadron spectrum [32] and the four definitions of the quark mass for each combination of heavy and light $\kappa$ value. We used the mass of the $D_{s}(1.9685$ $\mathrm{GeV}$ ) meson to determine the mass of the charm quark. To avoid a large extrapolation 


\begin{tabular}{ccc|ccc}
$\beta$ & $\kappa_{\text {sea }}$ & $a^{-1} \mathrm{GeV}$ & $\Lambda_{\overline{M S}} \mathrm{MeV}$ & $\alpha_{s}\left(a^{-1}\right)$ & $\alpha_{s}\left(\pi a^{-1}\right)$ \\
\hline 6.2 & 0.0 & 2.913 & 230 & 0.173 & 0.124 \\
6.0 & 0.0 & 2.119 & 219 & 0.191 & 0.133 \\
5.93 & 0.0 & 1.860 & 214 & 0.198 & 0.136 \\
5.2 & 0.1350 & 1.876 & 181 & 0.213 & 0.149
\end{tabular}

Table 2: Coupling constants using $\Lambda_{\overline{\mathrm{MS}}}$ from QCDSF-UKQCD collaboration 72 . The scale is set from $r_{0}=0.5$.

in the light quark mass we don't use the mass of the $D$ meson, as this is known to be problematic [74. As we only have one sea quark mass value, the chiral extrapolation is only done on the masses of the valence quarks. Hence, we obtain a result for the partially quenched charm mass.

In our earlier work [54] we used the spin average of the pseudo-scalar and vector masses of heavy-light mesons, because the pseudo-scalar-vector mass splitting is underestimated in the quenched QCD. However, we found it difficult to reliably estimate the $M_{2}$ meson mass for the vector meson, hence we now only use the pseudo-scalar meson.

The meson masses are first interpolated to the strange quark mass using a simple linear ansatz

$$
M\left(m_{l}\right)=a_{l}+b_{l} m_{l}
$$

where $m_{l}$ is the mass of the light quark. We denote this $M_{H s}$. The strange quark mass has already been determined by the UKQCD collaboration for these ensembles [30, 75]. The masses are then interpolated to the charm mass using

$$
M\left(m_{Q}\right)=a_{h}+b_{h} m_{Q}
$$

where $m_{Q}$ is the mass of the heavy quark. All the quark masses have corrections which scale with the quark mass, but the inverse hadron mass scales with quark mass, so it is unclear whether to plot $a m_{Q}$ vs. $1 / M_{H s}$ or vs. $M_{H s}$ [5]. We do the latter as we are interpolating in a finite range of $a m_{Q}$, where a polynomial in $a m_{Q}$ can be expanded in terms of $1 / a m_{Q}$. Hence we are treating the charm quark as a heavy light quark rather than a light heavy quark (where HQET based extrapolations would be appropriate). We consistently include any mass dependent renormalization factors in the definition of $m_{Q}$. Another option would have been to do the interpolation without the mass dependent renormalization factors, and then apply them after the fit.

We use capital $M$ for the meson masses and small $m$ for the quark masses. In our analysis we consider the options: $M_{1}$ versus $m_{0}, M_{1}$ versus $m_{p c a c}, M_{1}$ versus $m_{1}$, and $M_{2}$ versus $m_{2}$. The $m_{1}$ mass has a different mass behaviour to that of $m_{2}$ in the heavy quark limit, so it only really makes sense to match the $M_{2}$ mass with the $m_{2}$ mass.

\section{Results}

In this section we include some examples of the data fits that were performed, present results at fixed lattice spacing and consider the systematic error associated with $m_{c}\left(m_{c}\right)$ in the continuum limit. 


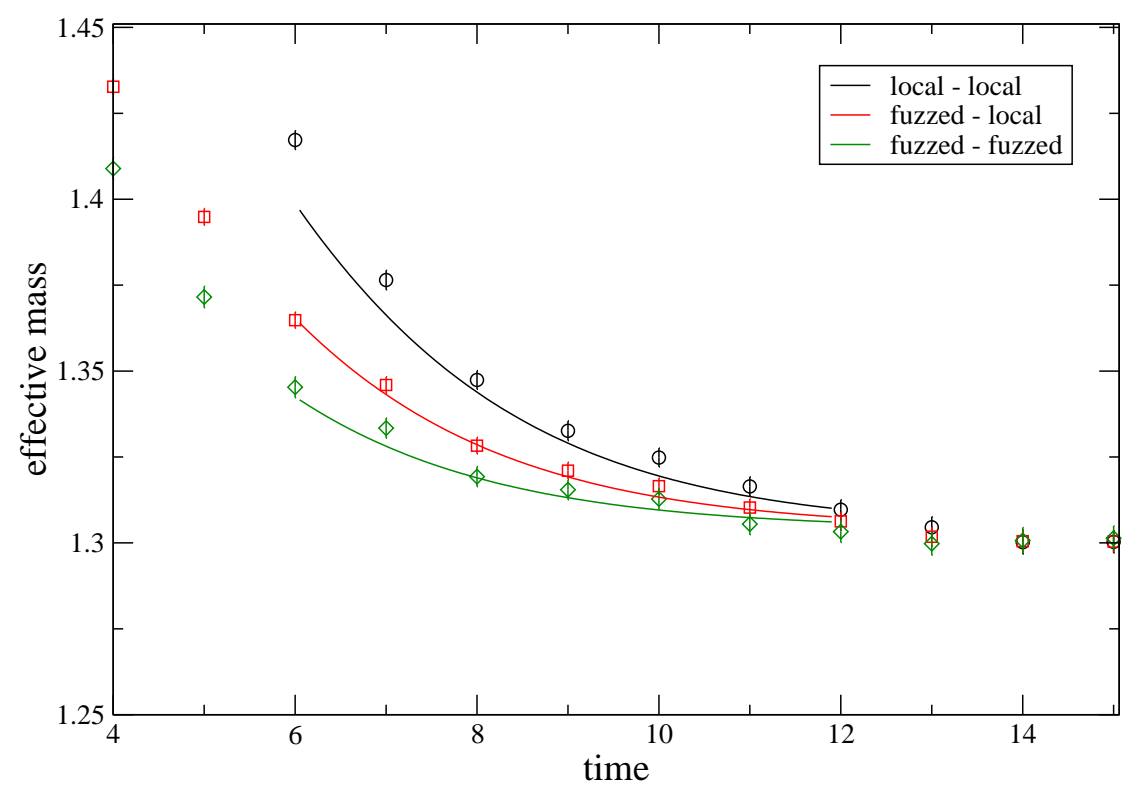

Figure 3: Example of a typical effective mass plot for the pseudo-scalar meson mass. The fit uses three exponentials to model data generated at $\beta=5.2, \kappa_{\mathrm{H}}=0.1130, \kappa_{\mathrm{L}}=0.1340$ and $\mathbf{p}=1,0,0$.

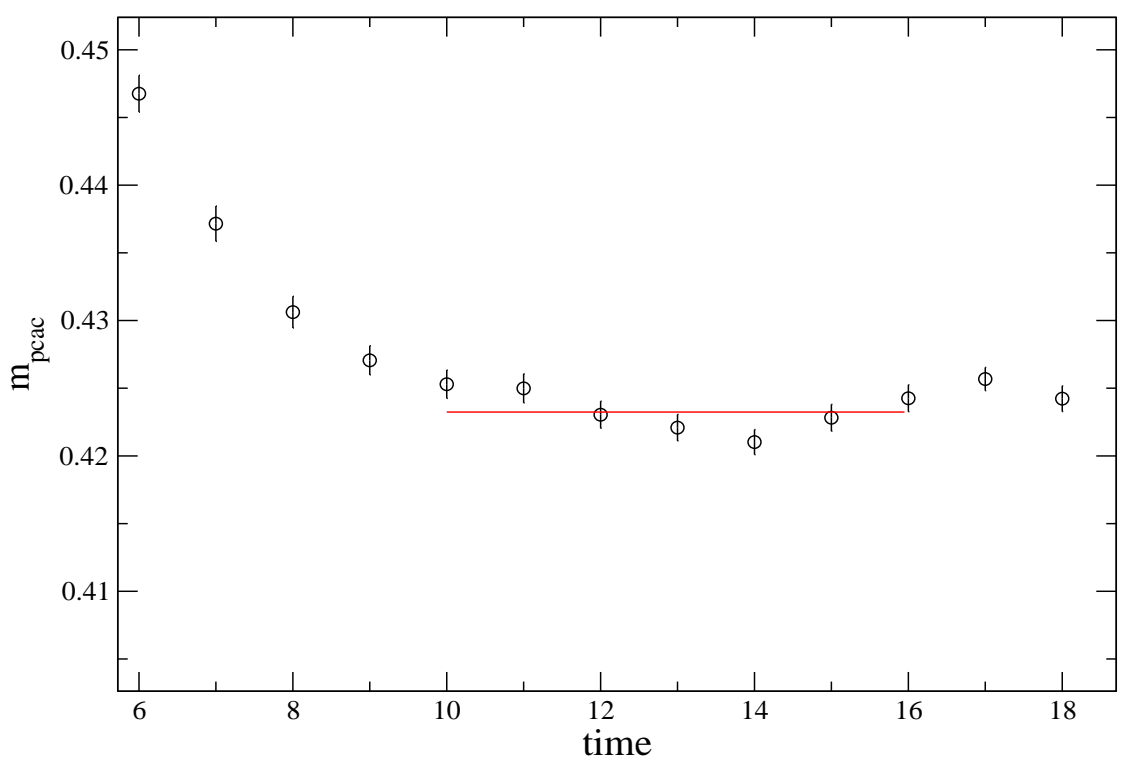

Figure 4: Example of a typical correlator plot for the PCAC mass. The fit uses a constant to model data generated at $\beta=5.2, \kappa_{\mathrm{H}}=0.1130, \kappa_{\mathrm{L}}=0.1340$ and $\mathbf{p}=0$.

\subsection{Examples of data fits}

An effective mass plot for the pseudo-scalar and a plot of the PCAC correlators are presented in figures 3 and 4 respectively. The heavy quark interpolation for $m_{A}$ on the matched 
ensembles is shown in figure 5 .

\subsection{Results at fixed lattice spacing}

As noted in many places (for example [30]), the determination of the $M_{2}$ meson from the dispersion relation in equation 3.15 produces masses with bigger errors than for the $M_{1}$ mass. So we use equation 3.20 to generate a perturbative estimate of the $M_{2}$ mass.

We test the approach in figures 6 and 7, where the perturbative estimate of $M_{2}$ is compared against the non-perturbative estimate from the dispersion relation. At the finer lattice spacing, $\beta=6.2$, all four definitions of the quark mass essentially agree.

\begin{tabular}{|c|c|c|c|c|}
\hline$\beta$ & $m_{\text {pcac }}$ & $m_{0}$ & $m_{1}$ & $m_{2}$ \\
\hline 5.2 & $1.327(4)_{-63}^{+36}$ & $0.952(1)_{-30}^{+16}$ & $1.247(3)_{-4}^{+20}$ & $1.266(3)_{-1}^{+6}$ \\
5.93 & $1.473(4)_{-64}^{+37}$ & $0.978(1)_{-28}^{+15}$ & $1.253(3)_{-5}^{+19}$ & $1.274(2)_{-0}^{+5}$ \\
6.0 & $1.439(4)_{-54}^{+31}$ & $1.025(2)_{-22}^{+14}$ & $1.265(4)_{-5}^{+21}$ & $1.283(3)_{-2}^{+9}$ \\
6.2 & $1.352(5)_{-50}^{+30}$ & $1.147(3)_{-12}^{+7}$ & $1.267(3)_{-7}^{+22}$ & $1.279(3)_{-5}^{+16}$ \\
\hline
\end{tabular}

Table 3: The mass of the charm quark in the $\overline{\mathrm{MS}}$ scheme at the charm mass scale, for different analysis techniques. We use $r_{0}=0.5 \mathrm{fm}$ as the central value and match at $\mu=2 / a$. The first error is statistical and the second is due to the perturbative matching.

Our results for the mass of the charm quark are presented in table 3 . The most striking point about the data is the differences between the results for the PCAC and vector masses. In quenched QCD the difference between the PCAC and vector quark masses is known to decrease as the continuum limit is taken [36, 6].

We have also used the non-perturbative value for $c_{A}$ which was recently published in 42] with $n_{f}=2$ sea quarks. This reduced the lattice quark masses by $10 \%$, consistent with the expectations in 42.

In figure 8 the dimensionless quantity $r_{0} M_{c}$ (where $M_{c}$ is the RG invariant mass) is plotted against the square of the lattice spacing. We compare our data to that of other groups at non-zero lattice spacing.

We used the convention for the renormalisation group invariant mass $\left(m_{\mathrm{RGI}}\right)$ used by the ALPHA collaboration 39.

$$
m_{\mathrm{RGI}}=\bar{m}\left(2 b_{0} g^{2}\right)^{-d_{0} / 2 b_{0}} \exp \left(\frac{1}{2 b_{0} g^{2}}\right) \exp \left(\int_{0}^{g} d \eta\left[\frac{1}{\beta(\eta)}+\frac{1}{b_{0} \eta^{3}}-\frac{b_{1}}{b_{0}^{2} \eta}\right]\right)
$$

This was different to the convention used in the RunDec package [71].

The large splitting between the vector and PCAC definition of the quark mass in our unquenched data is seen to be consistent with the quenched data of Rolf and Sint. However, the use of the nonperturbative renormalization factors by Rolf and Sint [6] complicates the comparison. Figure 8 shows that the agreement between the final answer for the charm quark mass from [5] and Rolf and Sint [6] is fortuitous. The final result from [5] was the average of the quark mass from the PCAC and vector currents. This is only a good estimate if the leading lattice spacing dependence from the PCAC and vector masses have opposite sign. 


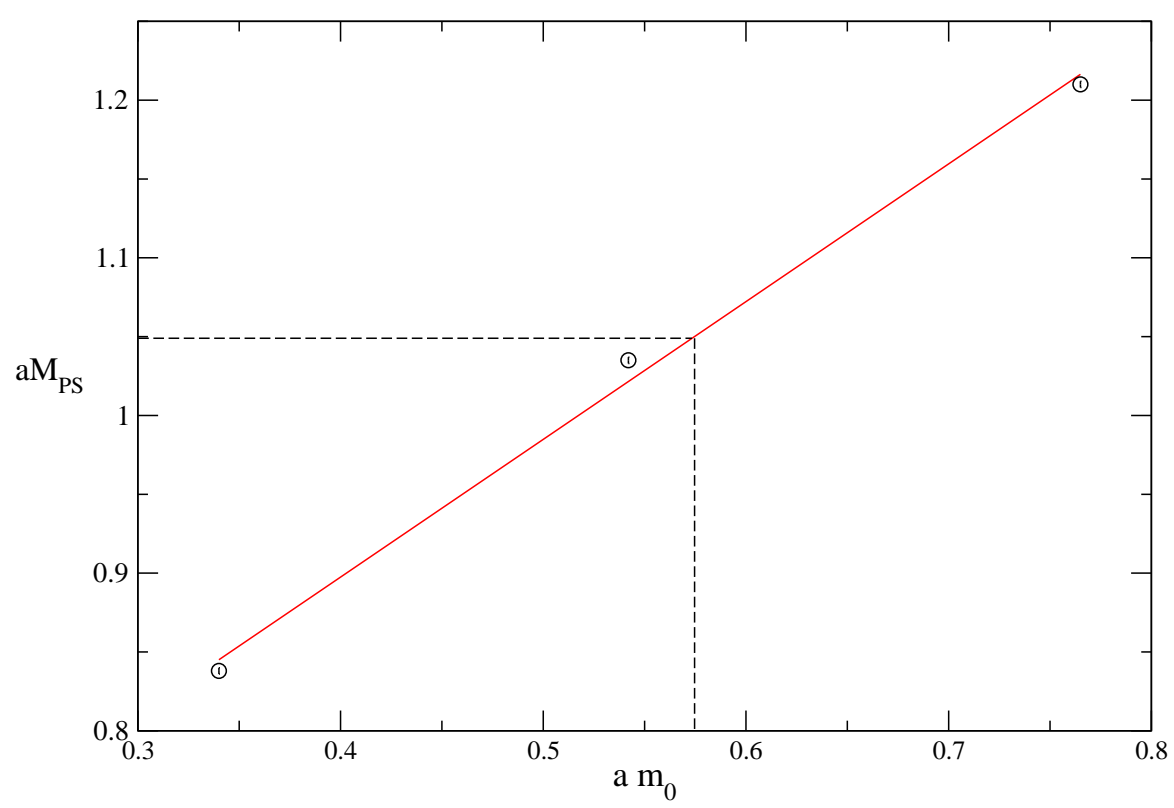

Figure 5: The hadron mass versus the bare quark mass for $\beta=5.2$. The dotted line highlights the physical meson mass in lattice units.

\subsection{Estimating the final systematic error}

To set the lattice spacing we use $r_{0}$ between 0.5 to $0.55 \mathrm{fm}$. The advantage of using $r_{0}$ to determine the lattice spacing is that it is relatively easy to determine the value of $r_{0}$ in lattice units. In [32], the results for $r_{0}$ in physical units, from a number of different calculations with unquenched Wilson like quarks were reviewed. All the results were between 0.5 and $0.55 \mathrm{fm}$ for $r_{0}$. The new results from unquenched calculations using improved staggered fermions [76, 77, 78] are finding $r_{0}$ values of $0.462(11)(4) \mathrm{fm}$. The use of the HPQCD [78] value for $r_{0}$ in the current generation of unquenched calculations with Wilson like fermions has been discussed in [23]. The QCDSF collaboration have used the nucleon mass, in unquenched clover calculations, to estimate $r_{0}=0.467 \mathrm{fm}$. However, it is difficult to do a reliable chiral extrapolation of the nucleon mass in the current generation of dynamical lattice QCD calculations, that use clover fermions [79], because of the size of the sea quark masses. For the data in this calculation we feel it is reasonable to use the estimate of $r_{0}$ between 0.5 and $0.55 \mathrm{fm}$.

We now consider the continuum limit of the charm quark mass using the data in table 3 . One issue concerns the dependence of the mass of the charm quark upon the lattice spacing. One simple model for the dependence of the charm mass at non-zero lattice spacing is

$$
m_{c}\left(m_{c}, a\right)=m_{c}\left(m_{c}\right)_{l}+\mathrm{s}_{l} a
$$




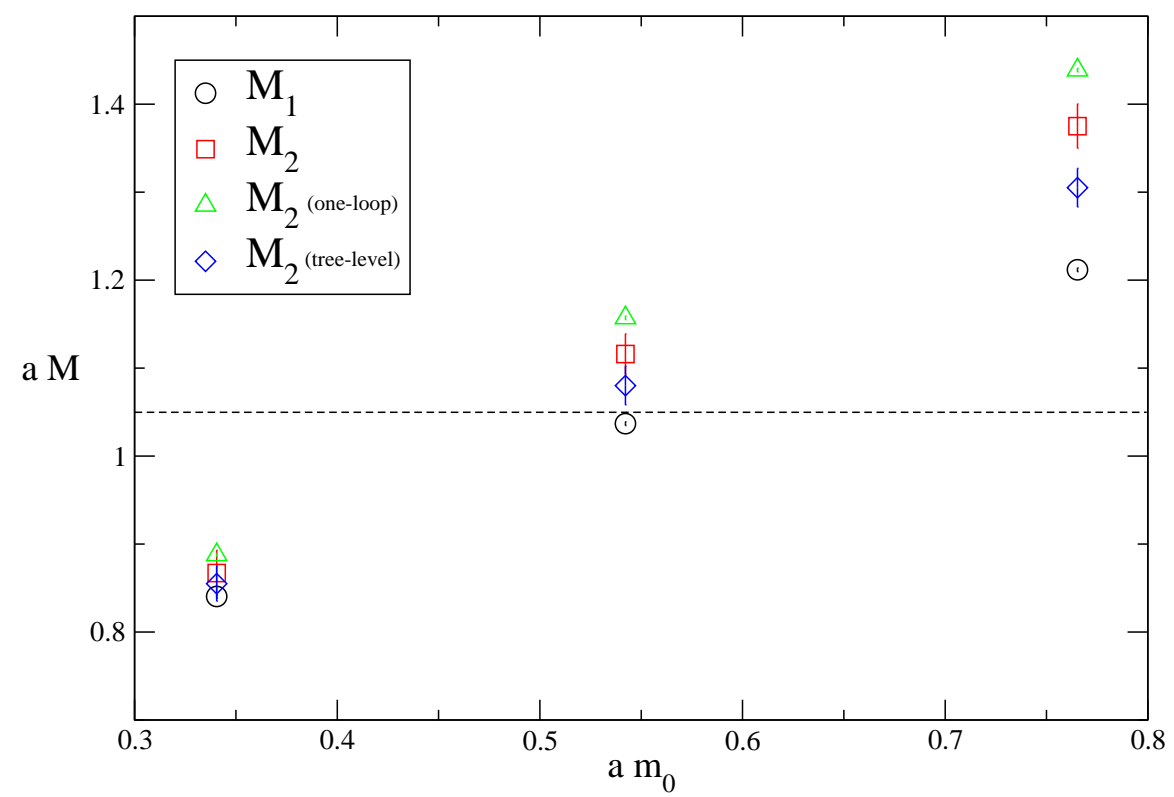

Figure 6: The $M_{1}$ and the $M_{2}$ mass computed from the dispersion relation, treel level and one loop in perturbation theory at $\beta=5.2$. The horizontal line represents the physical mass in lattice units.

The term linear in the lattice spacing comes from the use of improvement to one loop accuracy. We also tried a continuum extrapolation that was quadratic in the lattice spacing.

$$
m_{c}\left(m_{c}, a\right)=m_{c}\left(m_{c}\right)_{q}+\mathrm{s}_{q} a^{2}
$$

In table 4 we report the continuum extrapolation of the data in table 3 . In figures 9 and 10 the charm mass is plotted as a function of lattice spacing with the fitted continuum extrapolation using the model in equation 6.3 and 6.2 respectively.

\begin{tabular}{c|cc|cc} 
method & $m_{c}^{\overline{\mathrm{MS}}}\left(m_{c}\right)_{l} \mathrm{GeV}$ & $\mathrm{s}_{l} \mathrm{GeV}$ & $m_{c}\left(m_{c}\right)_{q} \mathrm{GeV}$ & $\mathrm{s}_{q} \mathrm{GeV}^{2}$ \\
\hline$m_{\text {pcac }}$ & $1.14(18)$ & $0.64(40)$ & $1.27(10)$ & $0.73(46)$ \\
$m_{0}$ & $1.45(5)$ & $-0.90(13)$ & $1.27(3)$ & $-1.0(2)$ \\
$m_{1}$ & $1.29(7)$ & $-0.07(15)$ & $1.28(4)$ & $-0.08(17)$ \\
$m_{2}$ & $1.30(4)$ & $-0.05(8)$ & $1.29(2)$ & $-0.05(9)$ \\
\hline
\end{tabular}

Table 4: Continuum limit of the mass of the charm quark mass in $\overline{\mathrm{MS}}$ at the mass of charm for different analysis techniques.

Figure 9 shows that a consistent continuum limit is obtained for all four definitions of quark mass if the extrapolations are done with equation 6.3, however it is difficult to give a rigorous argument in favour of this type of extrapolation. Figure 10 shows that the 


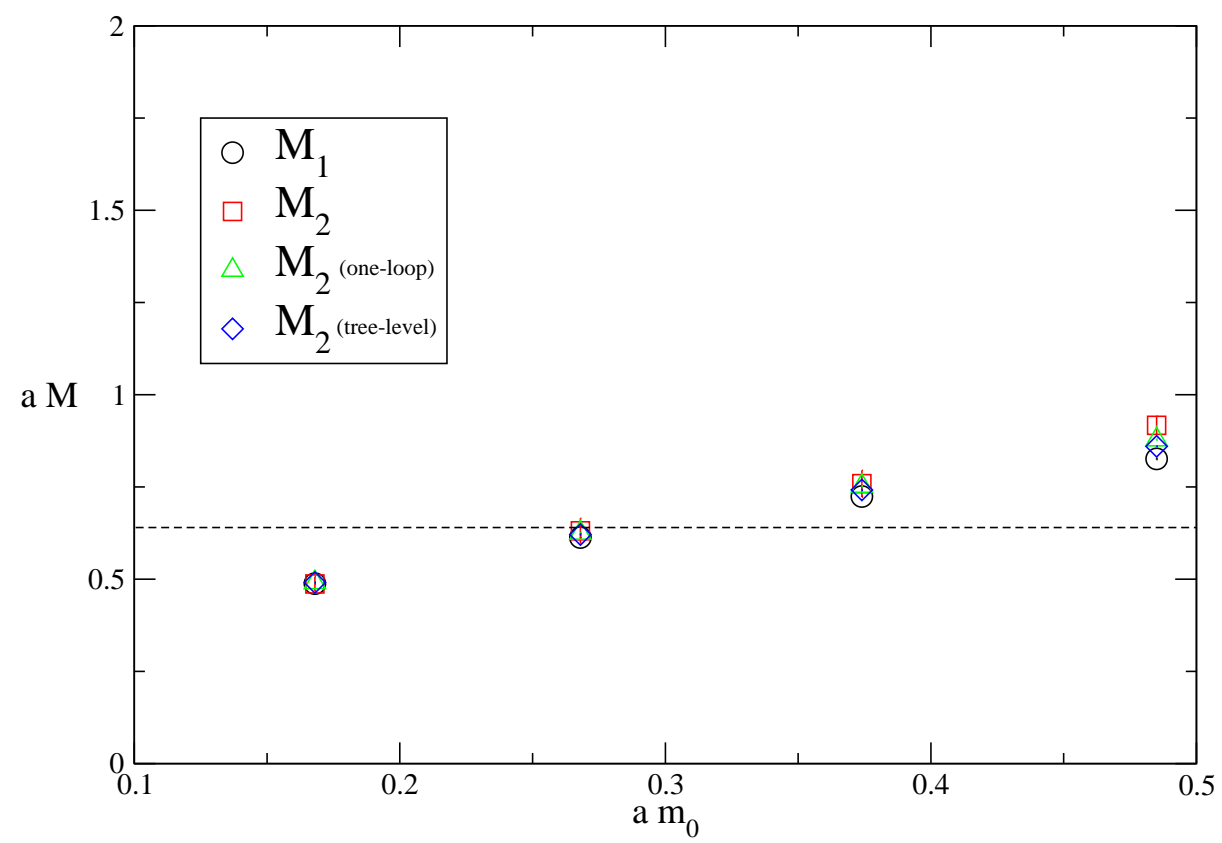

Figure 7: The $M_{1}$ and the $M_{2}$ mass computed from the dispersion relation, tree level and one loop in perturbation theory at $\beta=6.2$ quenched. The horizontal line represents the physical mass in lattice units.

continuum limit of the PCAC and vector masses is inconsistent with the FNAL result, if equation 6.2 is used to take the continuum limit. This fit looks poor and we speculate that the continuum extrapolation should be done with a combination of linear and quadratic dependence on the lattice spacing.

We also tried enforcing the same continuum limit for the vector and PCAC masses with fit parameters for both linear and quadratic terms in the lattice spacing. This gave $m_{c}^{\overline{\mathrm{MS}}}\left(m_{c}\right)=1.57 \pm 0.57 \mathrm{GeV}$. The fit is plotted in figure 10. Small $O(a)$ terms are not obtained, as the fit finds that both the $O(a)$ and $O\left(a^{2}\right)$ terms are large with opposite sign.

The situation may have become clearer, if we had used quenched QCD calculations at finer lattice spacings. However this would just repeat the work of Rolf and Sint [6]. To quote an unquenched result we need a formalism that produces the charm mass with a very weak dependence on the lattice spacing. As a check on our calculation, in table 5 we compare our results obtained with ALPHA formalism used by Rolf and Sint [6], with the results of Rolf and Sint. There is reasonable agreement between the two results.

The continuum extrapolation of the $m_{1}$ and $m_{2}$ masses is essentially consistent whether the numbers are extrapolated to the continuum limit either quadratically or linearly with lattice spacing. The extrapolation of heavy-light decay constants, obtained from calculations that use the FNAL formalism, were also insensitive to whether a linear or quadratic 


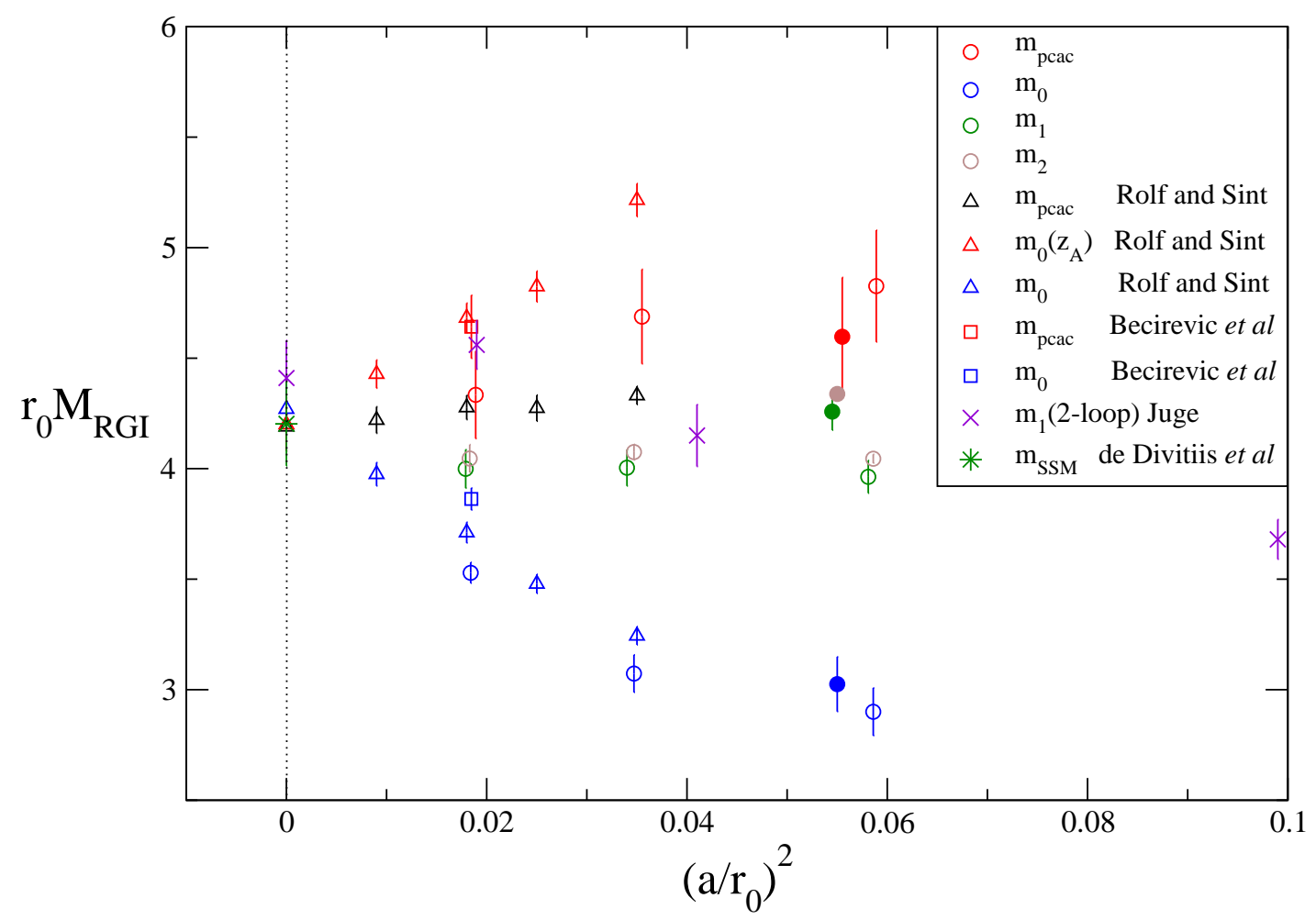

Figure 8: Recent data for the charm mass in the RGI scheme as a function of the lattice spacing. The filled circles are the results for $n_{f}=2$, all the other data is quenched.

\begin{tabular}{|c|cc|cc|}
\hline$\beta$ & \multicolumn{2}{|c|}{ This work } & \multicolumn{2}{c|}{ Rolf and Sint } \\
& $m_{0}^{R G I} \mathrm{GeV}$ & $m_{\text {pcac }}^{R G I} \mathrm{GeV}$ & $m_{0}^{R G I} \mathrm{GeV}$ & $m_{\text {pcac }}^{R G I} \mathrm{GeV}$ \\
\hline 6.0 & $3.273(39)$ & $4.430(52)$ & $3.224(41)$ & $4.331(59)$ \\
6.2 & $3.768(45)$ & $4.299(49)$ & $3.711(47)$ & $4.277(55)$ \\
\hline
\end{tabular}

Table 5: A comparison of the results from this paper with those from Rolf and Sint [6]. The renormalisation group invariant quark mass at charm for the vector and PCAC quark masses are reported using nonperturbative renormalisation factors.

extrapolation in lattice spacing was done [80].

For our quenched number we use the $m_{1}$ number in the continuum limit from a linear extrapolation in lattice spacing. We also quote an error of $10 \%$ to account for variations in lattice spacing determinations. Hence, our final result for mass of the charm quark in the continuum limit of quenched QCD is

$$
m_{c}\left(m_{c}\right)=1.29(7)(13) \mathrm{GeV}
$$

The first error is a combination of statistics and an estimate of the error due to only using perturbation theory to one loop order.

The data in table 3 do not show any clear pattern for the mass of the charm quark to differ between quenched and unquenched QCD at the fixed lattice spacing of $0.1 \mathrm{fm}$, with sea quarks with masses close to the strange value. Based on the scaling of the masses in 


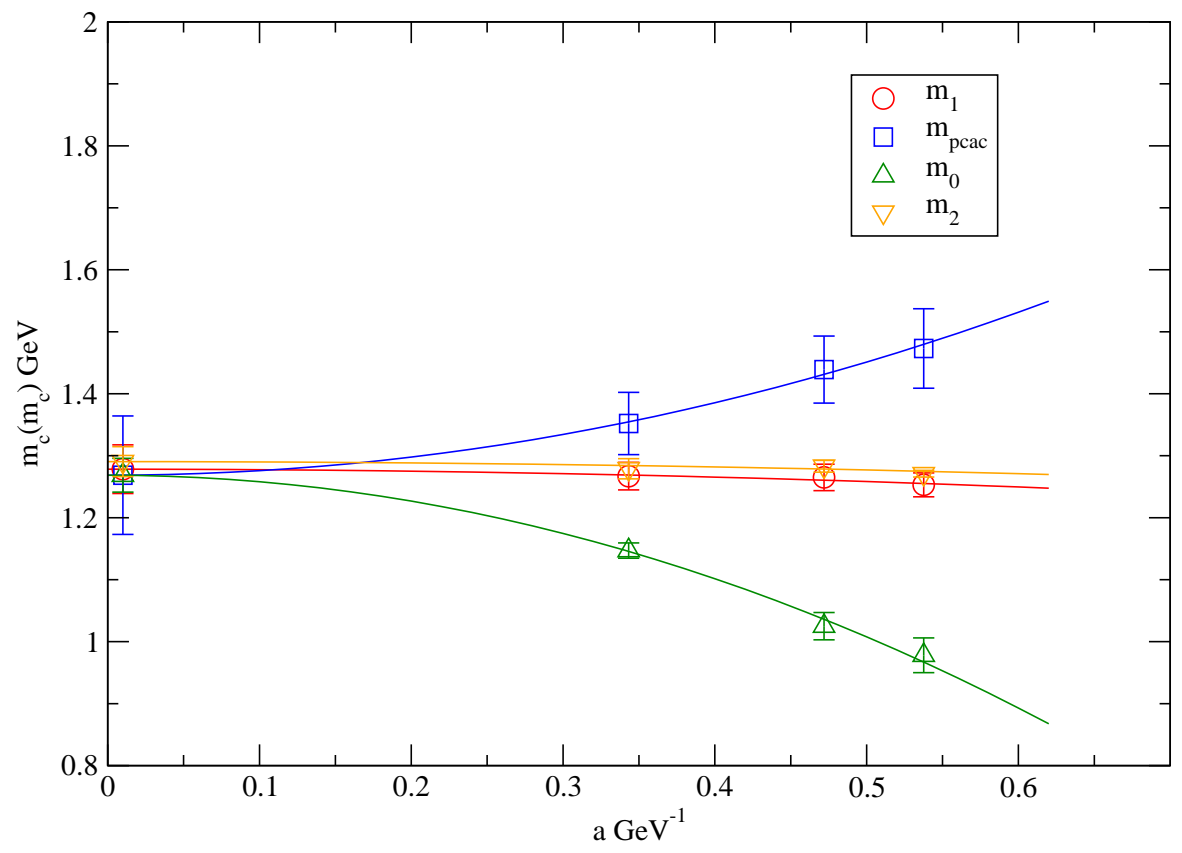

Figure 9: The quenched continuum limit of the scale invariant charm quark mass. The errors are from perturbative matching and statistics combined in quadrature. The curves are fits to a slope with $O\left(a^{2}\right)$ dependence only.

quenched QCD, for the unquenched data we use the value of the $m_{1}$ mass with a $10 \%$ error for determining the lattice spacing. Our best $n_{f}=2$ number is

$$
m_{c}\left(m_{c}\right)=1.247(3)_{-4}^{+20}(120) \mathrm{GeV}
$$

\section{Conclusions}

Our final result is $m_{c}^{\overline{\mathrm{MS}}}\left(m_{c}\right)=1.29(7)(13) \mathrm{GeV}$ in quenched QCD. We found that the ALPHA and FNAL formulations gave consistent numbers, after making some assumptions about the lattice spacing dependence. Our result is consistent with previous results from quenched QCD given in table 6 .

We have determined the mass of the charm quark in two flavour QCD at a lattice spacing of $0.1 \mathrm{fm}$ with a sea quark mass around strange value. We did not observe any unquenching errors.

The most important task for future unquenched lattice calculations is to control the lattice spacing errors in the mass of the charm quark. The large scaling violations found in the charm quark mass (see figure 8) with the improved clover action in quenched QCD can be controlled by the brute force method of using a lattice spacing of $0.05 \mathrm{fm}$. The timing 


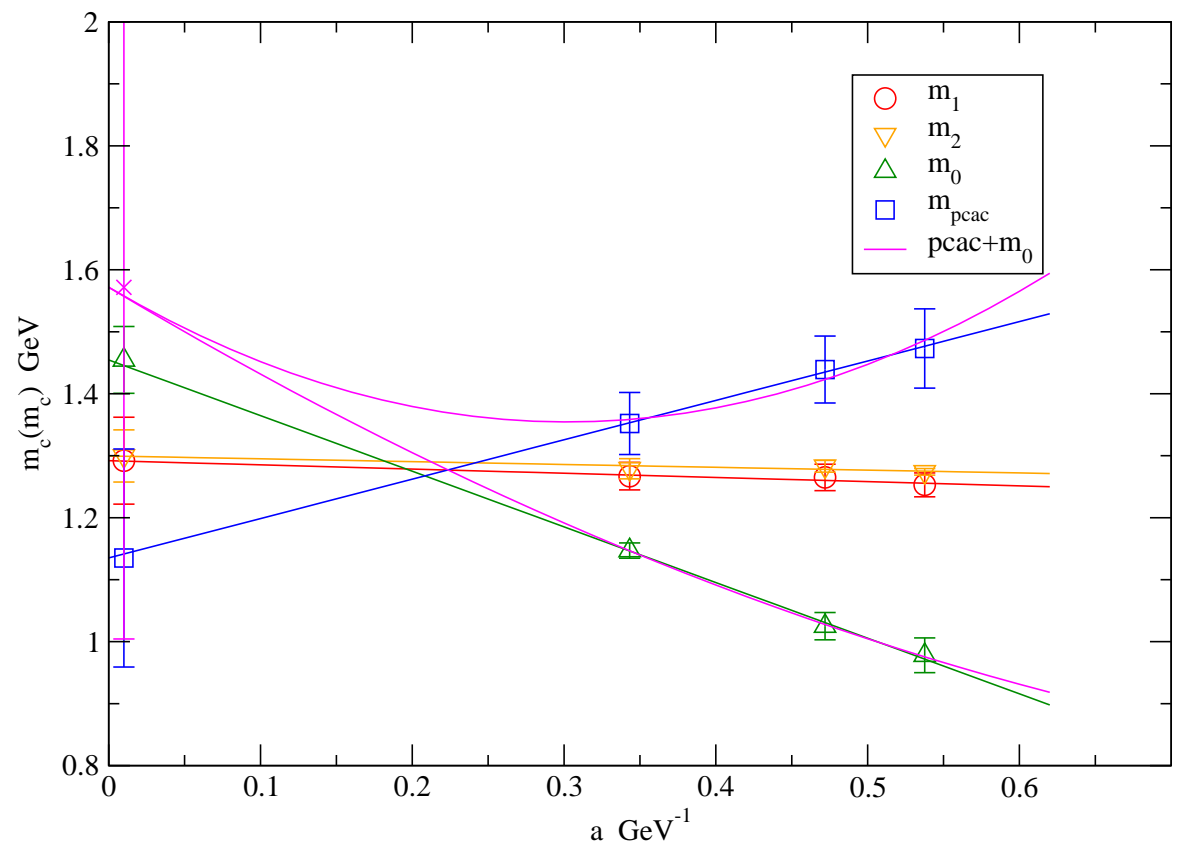

Figure 10: The quenched continuum limit of the scale invariant charm quark mass. The errors are from perturbative matching and statistics combined in quadrature. The curves are fits to a slope with $O(a)$ dependence only. The curve labelled by $p c a c+m_{0}$ is a fit where the pcac and vector quark masses are forced to have the same continuum limit.

estimates in [64, 65, 81] suggest that this approach will be not be easy for unquenched calculations because of the large computational cost in reducing the lattice spacing.

The FNAL formalism seems to have a better scaling behaviour than the ALPHA formalism for this data set. We note that one of the PCAC quark definitions used by Rolf and Sint also has a very weak lattice spacing dependence [6]. The development of better fermion actions for heavy quark calculations is clearly desirable $82,83,84,85,86]$.

Another important systematic error that must be reduced originates from matching the lattice renormalisation scheme onto the

\begin{tabular}{|cc|}
\hline Group & $m_{c}^{\overline{\mathrm{MS}}}\left(m_{c}\right) \mathrm{GeV}$ \\
\hline This work & $1.28(3)(13)$ \\
Becirevic et al. [0] & $1.26(4)(12)$ \\
Rolf and Sint [6] & $1.301(34)$ \\
Juge [7] & $1.27(5)$ \\
Kronfeld [8] & $1.33(8)$ \\
Hornbostel et al. [9] & $1.20(4)(11)(2)$ \\
de Divitiis et al. [10] & $1.319(28)$ \\
\hline
\end{tabular}

Table 6: Mass of the charm quark mass from various quenched lattice QCD calculations. continuum. As we discussed in section 4.2, there are many lattice techniques for reducing the error on the matching of the quark masses to the $\overline{\mathrm{MS}}$ scheme. Many of these techniques will benefit from unquenched data with finer lattice spacings. 


\section{Acknowledgements}

We thank Chris Michael for discussions. The lattice data was generated on the Cray T3D and T3E systems at EPCC supported by, EPSRC grant GR/K41663, PPARC grants GR/L22744 and PPA/G/S/1998/00777. We are grateful to the ULgrid project of the University of Liverpool for computer time.

\section{References}

[1] Particle Data Group Collaboration, S. Eidelman et. al., Review of particle physics, Phys. Lett. B592 (2004) 1.

[2] P. J. Bussey, Heavy flavour physics at hera: A survey, Int. J. Mod. Phys. A17 (2002) 1065-1110, hep-ph/0109254.

[3] L. Giusti, P. Hernandez, M. Laine, P. Weisz, and H. Wittig, A strategy to study the role of the charm quark in explaining the delta(i) =1/2 rule, JHEP 11 (2004) 016, hep-lat/0407007.

[4] N. Brambilla et. al., Heavy quarkonium physics, hep-ph/0412158.

[5] D. Becirevic, V. Lubicz, and G. Martinelli, Charm quark mass, Phys. Lett. B524 (2002) 115-122, hep-ph/0107124.

[6] ALPHA Collaboration, J. Rolf and S. Sint, A precise determination of the charm quark's mass in quenched qcd, JHEP 12 (2002) 007, hep-ph/0209255.

[7] K. J. Juge, The charm quark mass to two-loop order, Nucl. Phys. Proc. Suppl. 106 (2002) 847-849, http://arXiv.org/abs/hep-lat/0110131.

[8] A. S. Kronfeld, The charm quark's mass, Nucl. Phys. Proc. Suppl. 63 (1998) 311-313, hep-lat/9710007.

[9] NRQCD Collaboration, K. Hornbostel, Bottom and charm quark masses from lattice nrqcd, Nucl. Phys. Proc. Suppl. 73 (1999) 339-341, hep-lat/9809177.

[10] G. M. de Divitiis, M. Guagnelli, R. Petronzio, N. Tantalo, and F. Palombi, Heavy quark masses in the continuum limit of lattice qcd, Nucl. Phys. B675 (2003) 309-332, hep-lat/0305018.

[11] P. B. Mackenzie, Recent lattice results on the light quark masses, Nucl. Phys. Proc. Suppl. 53 (1997) 23-29, http://arXiv.org/abs/hep-ph/9609261.

[12] B. J. Gough et. al., The light quark masses from lattice gauge theory, Phys. Rev. Lett. 79 (1997) 1622-1625, http://arXiv.org/abs/hep-ph/9610223.

[13] CP-PACS Collaboration, A. Ali Khan et. al., Dynamical quark effects on light quark masses, Phys. Rev. Lett. 85 (2000) 4674-4677, hep-lat/0004010.

[14] HPQCD+MILC+UKQCD Collaboration, C. Aubin et. al., First determination of the strange and light quark masses from full lattice qcd, Phys. Rev. D70 (2004) 031504, hep-lat/0405022.

[15] HPQCD Collaboration, Q. Mason, H. D. Trottier, R. Horgan, C. T. H. Davies, and G. P. Lepage, High-precision determination of the light-quark masses from realistic lattice qcd, hep-ph/0511160. 
[16] QCDSF Collaboration, M. Gockeler et. al., Determination of light and strange quark masses from full lattice qcd, hep-ph/0409312.

[17] D. Becirevic et. al., Non-perturbatively renormalised light quark masses from a lattice simulation with $n(f)=2$, Nucl. Phys. B734 (2006) 138-155, hep-lat/0510014.

[18] ALPHA Collaboration, M. Della Morte et. al., Non-perturbative quark mass renormalization in two-flavor qcd, hep-lat/0507035.

[19] M. Gockeler et. al., Estimating the unquenched strange quark mass from the lattice axial ward identity, hep-lat/0601004.

[20] C. T. H. Davies et. al., A new determination of $m(b)$ using lattice qcd, Phys. Rev. Lett. 73 (1994) 2654-2657, http://arXiv.org/abs/hep-lat/9404012.

[21] V. Gimenez, L. Giusti, G. Martinelli, and F. Rapuano, Nnlo unquenched calculation of the $b$ quark mass, JHEP 03 (2000) 018, hep-lat/0002007.

[22] F. Di Renzo and L. Scorzato, The $n(f)=2$ residual mass in perturbative lattice-hqet for an improved determination of the $m(b)(m s-b a r)(m(b)(m s-b a r))$, hep-lat/0408015.

[23] UKQCD Collaboration, C. McNeile, C. Michael, and G. Thompson, An unquenched lattice qcd calculation of the mass of the bottom quark, hep-lat/0408025.

[24] V. Lubicz, Quark masses on the lattice: Light and heavy, Nucl. Phys. Proc. Suppl. 94 (2001) 116-129, http://arXiv.org/abs/hep-lat/0012003.

[25] H. Wittig, Chiral effective lagrangian and quark masses, Nucl. Phys. Proc. Suppl. 119 (2003) 59-70, hep-lat/0210025.

[26] P. E. L. Rakow, Progress towards finding quark masses and the qcd scale lambda from the lattice, Nucl. Phys. Proc. Suppl. 140 (2005) 34-47, hep-lat/0411036.

[27] A. S. Kronfeld, Heavy quarks and lattice qcd, Nucl. Phys. Proc. Suppl. 129 (2004) 46-59, hep-lat/0310063.

[28] S. Hashimoto and T. Onogi, Heavy quarks on the lattice, Ann. Rev. Nucl. Part. Sci. 54 (2004) 451-486, hep-ph/0407221.

[29] UKQCD Collaboration, K. C. Bowler et. al., Quenched qcd with o(a) improvement. $i$ : The spectrum of light hadrons, Phys. Rev. D62 (2000) 054506, hep-lat/9910022.

[30] UKQCD Collaboration, K. C. Bowler et. al., Decay constants of $b$ and d mesons from non-perturbatively improved lattice qcd, Nucl. Phys. B619 (2001) 507-537, http://arXiv.org/abs/hep-lat/0007020.

[31] UKQCD Collaboration, C. R. Allton et. al., Effects of non-perturbatively improved dynamical fermions in qcd at fixed lattice spacing, Phys. Rev. D65 (2002) 054502, http://arXiv.org/abs/hep-lat/0107021.

[32] UKQCD Collaboration, A. Dougall, R. D. Kenway, C. M. Maynard, and C. McNeile, The spectrum of d/s mesons from lattice qcd, Phys. Lett. B569 (2003) 41-44, hep-lat/0307001.

[33] UKQCD Collaboration, C. McNeile and C. Michael, An estimate of the flavour singlet contributions to the hyperfine splitting in charmonium, hep-lat/0402012.

[34] UKQCD Collaboration, P. Boyle, A novel gauge invariant multi-state smearing technique, J. Comput. Phys. 179 (2002) 349-370, http://arXiv.org/abs/hep-lat/9903033. 
[35] UKQCD Collaboration, P. Lacock, A. McKerrell, C. Michael, I. M. Stopher, and P. W. Stephenson, Efficient hadronic operators in lattice gauge theory, Phys. Rev. D51 (1995) 6403-6410, hep-lat/9412079.

[36] R. Gupta and T. Bhattacharya, Light quark masses from lattice qcd, Phys. Rev. D55 (1997) 7203-7217, hep-lat/9605039.

[37] K. Jansen et. al., Non-perturbative renormalization of lattice qcd at all scales, Phys. Lett. B372 (1996) 275-282, hep-lat/9512009.

[38] M. Luscher, S. Sint, R. Sommer, and P. Weisz, Chiral symmetry and o(a) improvement in lattice qcd, Nucl. Phys. B478 (1996) 365-400, http://arXiv.org/abs/hep-lat/9605038.

[39] ALPHA Collaboration, S. Capitani, M. Luscher, R. Sommer, and H. Wittig, Non-perturbative quark mass renormalization in quenched lattice qcd, Nucl. Phys. B544 (1999) 669-698, hep-lat/9810063.

[40] M. Luscher and P. Weisz, $O(a)$ improvement of the axial current in lattice qcd to one-loop order of perturbation theory, Nucl. Phys. B479 (1996) 429-458, http://arXiv.org/abs/hep-lat/9606016.

[41] S. Sint and P. Weisz, Further results on o(a) improved lattice qcd to one-loop order of perturbation theory, Nucl. Phys. B502 (1997) 251-268, hep-lat/9704001.

[42] M. Della Morte, R. Hoffmann, and R. Sommer, Non-perturbative improvement of the axial current for dynamical wilson fermions, JHEP 03 (2005) 029, hep-lat/0503003.

[43] G. P. Lepage and P. B. Mackenzie, On the viability of lattice perturbation theory, Phys. Rev. D48 (1993) 2250-2264, hep-lat/9209022].

[44] T. Bhattacharya, R. Gupta, W.-J. Lee, and S. R. Sharpe, Order a improved renormalization constants, Phys. Rev. D63 (2001) 074505, http://arXiv.org/abs/hep-lat/0009038.

[45] UKQCD Collaboration, S. Collins, C. T. H. Davies, G. P. Lepage, and J. Shigemitsu, A nonperturbative determination of the o(a) improvement coefficient $c(a)$ and the scaling of $f($ pi) and $m$ (ms-bar), Phys. Rev. D67 (2003) 014504, hep-lat/0110159].

[46] A. X. El-Khadra, A. S. Kronfeld, and P. B. Mackenzie, Massive fermions in lattice gauge theory, Phys. Rev. D55 (1997) 3933-3957, hep-lat/9604004.

[47] C. R. Allton et. al., Quark masses from lattice qcd at the next-to-leading order, Nucl. Phys. B431 (1994) 667-685, hep-ph/9406263.

[48] M. Gockeler et. al., Scaling of non-perturbatively o(a) improved wilson fermions: Hadron spectrum, quark masses and decay constants, Phys. Rev. D57 (1998) 5562-5580, http://arXiv.org/abs/hep-lat/9707021.

[49] J. Harada, S. Hashimoto, A. S. Kronfeld, and T. Onogi, Perturbative calculation of o(a) improvement coefficients, Phys. Rev. D67 (2003) 014503, hep-lat/0208004.

[50] B. P. G. Mertens, A. S. Kronfeld, and A. X. El-Khadra, The self energy of massive lattice fermions, Phys. Rev. D58 (1998) 034505, hep-lat/9712024.

[51] E. Eichten and B. Hill, Renormalization of heavy - light bilinears and $f(b)$ for wilson fermions, Phys. Lett. B240 (1990) 193.

[52] S. Groote and J. Shigemitsu, One-loop self energy and renormalization of the speed of light for some anisotropic improved quark actions, Phys. Rev. D62 (2000) 014508, hep-lat/0001021. 
[53] C. T. H. Davies and B. A. Thacker, Renormalization of bilinears in nonrelativistic qcd, Phys. Rev. D48 (1993) 1329-1336.

[54] UKQCD Collaboration, A. Dougall, C. M. Maynard, and C. McNeile, The mass of the charm quark from unquenched lattice qcd at fixed lattice spacing, Nucl. Phys. Proc. Suppl. 129 (2004) 170-172, hep-lat/0309081.

[55] UKQCD Collaboration, A. Dougall, C. M. Maynard, and C. McNeile, The charm quark mass with dynamical fermions, Nucl. Phys. Proc. Suppl. 140 (2005) 428-430, hep-lat/0409089.

[56] M. Luscher, Advanced lattice qcd, hep-lat/9802029.

[57] S. Sint, Non-perturbative renormalization in lattice field theory, Nucl. Phys. Proc. Suppl. 94 (2001) 79-94, hep-lat/0011081.

[58] R. Sommer, Non-perturbative renormalization of hqet and qcd, Nucl. Phys. Proc. Suppl. 119 (2003) 185-197, hep-lat/0209162.

[59] H. D. Trottier, Higher-order perturbation theory for highly-improved actions, Nucl. Phys. Proc. Suppl. 129 (2004) 142-148, hep-lat/0310044.

[60] UKQCD Collaboration, R. Horsley, Determining g(a) using non-perturbatively o(a) improved wilson fermions, Nucl. Phys. Proc. Suppl. 94 (2001) 307-310, http://arXiv.org/abs/hep-lat/0010059.

[61] ALPHA Collaboration, F. Knechtli et. al., Running quark mass in two flavor qcd, http://arXiv.org/abs/hep-lat/0209025.

[62] QCDSF-UKQCD Collaboration, T. Bakeyev et. al., Non-perturbative renormalisation and improvement of the local vector current for quenched and unquenched wilson fermions, Phys. Lett. B580 (2004) 197-208, hep-lat/0305014.

[63] M. Della Morte, R. Hoffmann, F. Knechtli, R. Sommer, and U. Wolff, Non-perturbative renormalization of the axial current with dynamical wilson fermions, hep-lat/0505026.

[64] K. Jansen, Actions for dynamical fermion simulations: Are we ready to go?, Nucl. Phys. Proc. Suppl. 129 (2004) 3-16, hep-lat/0311039.

[65] A. D. Kennedy, Algorithms for lattice qcd with dynamical fermions, Nucl. Phys. Proc. Suppl. 140 (2005) 190-203, [hep-lat/0409167].

[66] ALPHA Collaboration, M. Guagnelli et. al., Non-perturbative results for the coefficients $b(m)$ and $b(a)-b(p)$ in o(a) improved lattice qcd, Nucl. Phys. B595 (2001) 44-62, http://arXiv.org/abs/hep-lat/0009021.

[67] ALPHA and UKQCD Collaboration, J. Garden, J. Heitger, R. Sommer, and H. Wittig, Precision computation of the strange quark's mass in quenched qcd, Nucl. Phys. B571 (2000) 237, hep-lat/9906013.

[68] H.-W. Lin, Non-perturbative renormalization and the fermilab action, Nucl. Phys. Proc. Suppl. 129 (2004) 429-431, hep-lat/0310060.

[69] H.-W. Lin, Non-perturbative determination of heavy quark action coefficients, hep-lat/0409085.

[70] ALPHA Collaboration, J. Heitger and R. Sommer, Non-perturbative heavy quark effective theory, JHEP 02 (2004) 022, hep-lat/0310035. 
[71] K. G. Chetyrkin, J. H. Kuhn, and M. Steinhauser, Rundec: A mathematica package for running and decoupling of the strong coupling and quark masses, Comput. Phys. Commun. 133 (2000) 43-65, hhttp://arXiv.org/abs/hep-ph/0004189].

[72] QCDSF-UKQCD Collaboration, S. Booth et. al., Determination of lambda(ms-bar) from quenched and $n(f)=2$ dynamical qcd, Phys. Lett. B519 (2001) 229-237, http://arXiv.org/abs/hep-lat/0103023.

[73] R. Gupta, T. Bhattacharya, and S. Sharpe, Matrix elements of 4-fermion operators with quenched wilson fermions, Phys. Rev. D55 (1997) 4036-4054, hep-lat/9611023.

[74] X. H. Guo and A. W. Thomas, Chiral extrapolation of lattice data for the hyperfine splittings of heavy mesons, Phys. Rev. D65 (2002) 074019, http://arXiv.org/abs/hep-ph/0112040.

[75] UKQCD Collaboration, D. J. Hepburn, Light quark masses from ukqcd's dynamical simulations with o(a)-improved wilson fermions, hep-lat/0209036.

[76] C. Aubin et. al., Light hadrons with improved staggered quarks: Approaching the continuum limit, Phys. Rev. D70 (2004) 094505, hep-lat/0402030.

[77] M. Wingate, C. T. H. Davies, A. Gray, G. P. Lepage, and J. Shigemitsu, The $b / s$ and $d / s$ decay constants in 3 flavor lattice qcd, Phys. Rev. Lett. 92 (2004) 162001, hep-ph/0311130.

[78] A. Gray et. al., The upsilon spectrum and $m(b)$ from full lattice qcd, hep-lat/0507013.

[79] S. R. Beane, In search of the chiral regime, Nucl. Phys. B695 (2004) 192-198, hep-lat/0403030.

[80] A. X. El-Khadra, A. S. Kronfeld, P. B. Mackenzie, S. M. Ryan, and J. N. Simone, $B$ and $d$ meson decay constants in lattice qcd, Phys. Rev. D58 (1998) 014506, hep-ph/9711426.

[81] UKQCD Collaboration, H. Wittig, Cost of dynamical quark simulations: O(a) improved wilson fermions, Nucl. Phys. Proc. Suppl. 106 (2002) 197-198, hep-lat/0203021.

[82] Z. Sroczynski, A. X. El-Khadra, A. S. Kronfeld, P. B. Mackenzie, and J. N. Simone, First results from the asymmetric o(a) improved fermilab action, Nucl. Phys. Proc. Suppl. 83 (2000) 971-973.

[83] M. di Pierro et. al., Properties of charmonium in lattice qcd with 2+1 flavors of improved staggered sea quarks, Nucl. Phys. Proc. Suppl. 129 (2004) 340-342, hep-lat/0310042.

[84] M. B. Oktay, A. X. El-Khadra, A. S. Kronfeld, and P. B. Mackenzie, A more improved lattice action for heavy quarks, Nucl. Phys. Proc. Suppl. 129 (2004) 349-351, hep-lat/0310016.

[85] TrinLat Collaboration, G. Burgio, A. Feo, M. J. Peardon, and S. M. Ryan, Gauge theories on a 2+2 anisotropic lattice, Phys. Rev. D67 (2003) 114502, hep-lat/0303005.

[86] CP-PACS Collaboration, Y. Kayaba et. al., Charmed meson spectra and decay constants with one-loop o(a) improved relativistic heavy quark action, hep-lat/0409086. 